The Impact of Large-Scale Surveys on Pulsating Star Research

ASP Conference Series, Vol. 203, 2000

L. Szabados \& D. W. Kurtz, eds.

\title{
Nonlinear Pulsations of Luminous Blue Variables
}

\author{
Ernst A. Dorfi \\ Institut für Astronomie, Universität Wien, Türkenschanzstraße 17, \\ A-1180 Wien, Austria \\ Michael U. Feuchtinger \\ Physics Department, University of Florida, PO Box 118440 , \\ Gainesville, 32611, USA \\ Alfred Gautschy \\ Astronomisches Institut der Universität Basel, Venusstrasse 7, \\ CH-4102 Binningen, Switzerland
}

\begin{abstract}
We present the results of various nonlinear radial pulsation calculations of LBVs with different luminosity to mass ratios. Depending on the stellar parameters, most of these objects undergo strongly nonadiabatic pulsations which can also modify their stellar atmospheres. In some cases part of the kinetic energy of the motions is transferred by shock waves into the atmospheric layers leading to an overall decrease or increase of the stellar radius. This results in different mean density accompanied by a change of the pulsational behavior which can be compared to the results of a linear stability analysis. We also find that regular pulsations can occur around a different equilibrium than the initial configuration which has been determined by stellar evolution calculations. In cases of even higher luminosity to mass ratios the linear stability analysis predicts several unstable modes and the nonlinear computations can exhibit rather irregular light curves. Due to the large non-adiabaticity of the pulsation the corresponding velocity curves still remain more regular, but show increasing amplitudes until the energy stored is released. The pulsations are far from equilibrium conditions and may also lead to a pulsationally driven mass loss for certain stellar parameters.
\end{abstract}

\section{Introduction}

Luminous Blue Variables (LBVs) are among stars the most luminous stars, with luminosities up to $10^{6} L_{\odot}$ and masses up to $100 M_{\odot}$. Due to their highly unusual stellar spectra most of the physical parameters, like the effective temperature, cannot be well defined; however, plotted in the HRD all LBVs are located below the Humphreys-Davidson limit. Concerning their high luminosities the radiation pressure plays a dominant role putting these stars near the Eddington limit. The observed light curves show irregular variations on different time scales ranging from minutes to large outbursts of several months. The LBVs are also char- 
acterized by emission lines and $\mathrm{P}$ Cygni profiles indicating a pronounced mass loss $\left(\dot{M} \gtrsim 10^{-4} M_{\odot} \mathrm{yr}^{-1}\right)$ which may occur mainly during such large eruptions. This mass loss can be seen as circumstellar nebulae associated with essentially all LBVs. Taking the inferred masses and luminosities into account it is clear that such an active phase of about $\Delta t \lesssim 10^{5} \mathrm{yr}$ will last only for a short time limiting, e.g., the number of known LBVs to about thirty within nine galaxies (cf. Nota \& Lamers 1997 for recent proceedings on LBVs). Collecting this information we see that LBVs are described by non-equilibrium as well as nonlinear processes which have to be studied by numerical computations.

The observed LBV variabilities might serve as an independent class of markers of the extragalactic distance scale if their pulsational and eruption properties are better understood and can be inferred, e.g., from theoretical computations.

\section{Nonlinear Pulsation Calculations}

To study LBV pulsations we have performed nonlinear calculations using the Vienna pulsation code (see Dorfi 1998) which solves the full system of radiation hydrodynamics on an adaptive grid in conservation form. The gray radiative transfer involves also the computation of the variable Eddington factor (Yorke 1980 ) to close the system of equations accounting for the extended atmospheres. The simulations presented here are purely radiative models without the inclusion of convective energy transport (see Feuchtinger 1999).

Table 1. Parameters of LBV models.

\begin{tabular}{ccccccrr}
\hline$M$ & $L$ & $T_{\text {eff }}$ & $R$ & $P_{\text {lin }}$ & $P_{\text {nl }}$ & $\Delta T$ & $\Delta R / R$ \\
{$\left[M_{\odot}\right]$} & {$\left[10^{5} L_{\odot}\right]$} & {$\left[10^{3} \mathrm{~K}\right]$} & $R_{\odot}$ & {$[\mathrm{d}]$} & {$[\mathrm{d}]$} & {$[\mathrm{K}]$} & $R_{\odot}$ \\
\hline 22 & 3.2 & 31.6 & 18.7 & 0.98 & 1.78 & -1400 & 2.3 \\
60 & 7.0 & 12.0 & 193.4 & & 6.21 & 140 & -0.4 \\
60 & 9.0 & 18.0 & 97.5 & & 4.15 & 3000 & -22.6 \\
90 & 13.2 & 35.9 & 29.6 & 0.88 & 1.13 & -2900 & 3.3 \\
\hline
\end{tabular}

The pulsational behavior of four models with initial values of mass $M$, luminosity $L$ and effective temperature $T_{\text {eff }}$ is summarized in Table 1 . These LBVs exhibit rather regular pulsations with a nonlinear period $P_{\mathrm{nl}}$ around a new effective temperature with $\Delta T$ and a new mean radius with $\Delta R / R$ compared to the equilibrium values given by the static stellar structure equations. In two cases the one linear unstable period $P_{\text {lin }}$ is also included. Such small scale variations are also found in the observations as so-called microvariability. Some simulations with more violent pulsations have been presented in Dorfi (1999), but also those LBVs with more unstable modes do not show a pulsational driven mass loss. As inferred from Table 1, depending on the initial physical parameters we find both expanding as well as contracting stellar envelopes. Since only a part of the stellar envelope is affected by the waves, the period changes are not simply related by the variation of the mean density. 

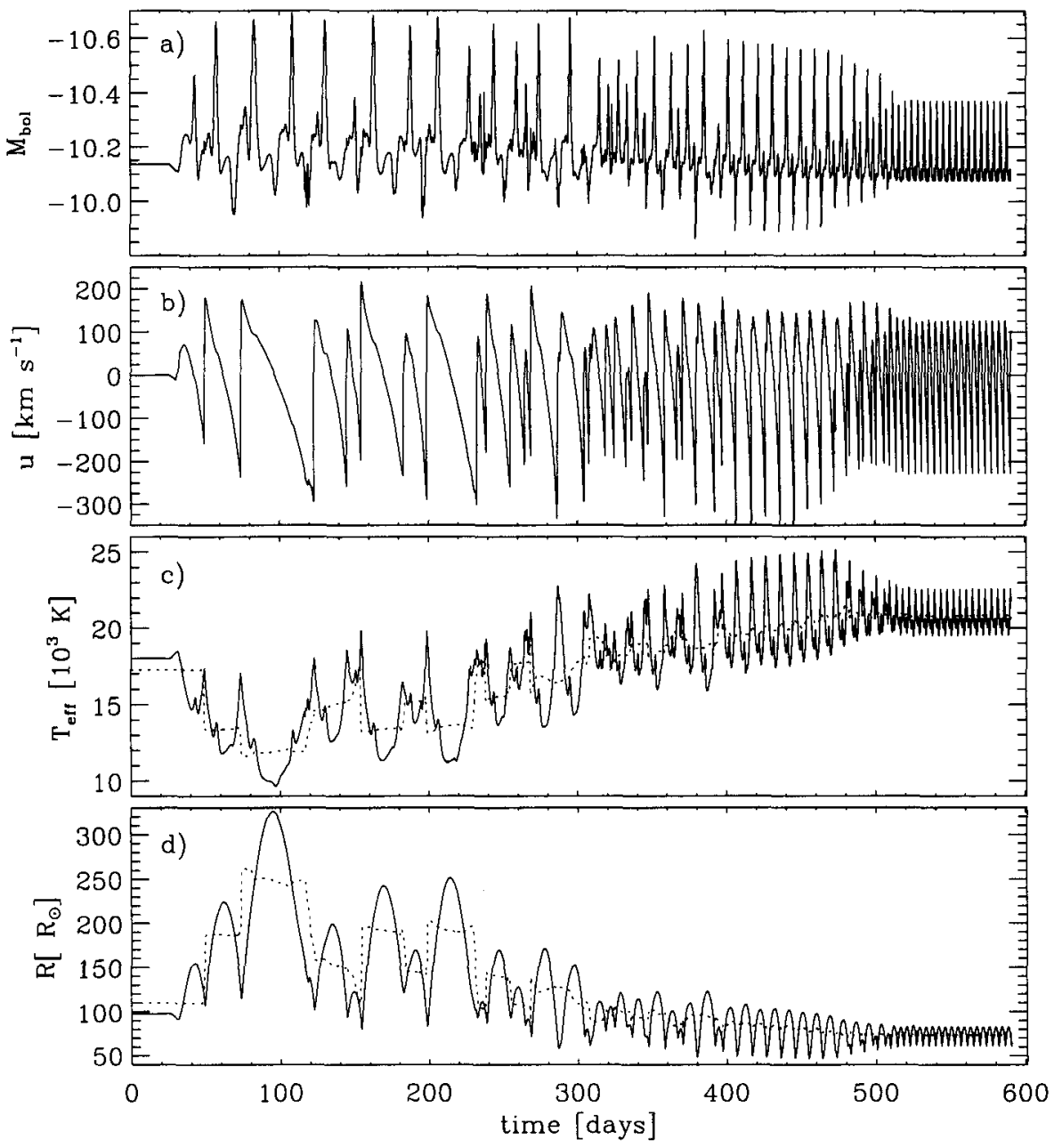

Figure 1. The pulsation of an LBV with $M=60 M_{\odot}, L=9 \cdot 10^{5} L_{\odot}$ and $T_{\text {eff }}=18000 \mathrm{~K}$ showing the transition into a different equilibrium. As the radius gets smaller (see Fig. 1d) mean effective temperature (Fig. 1c) has to increase to maintain the total luminosity. The dotted line depicts the running mean over 1000 time steps. The temporal evolution over these first $600 \mathrm{~d}$ is small compared to the thermal relaxation time scale of about $10 \mathrm{yr}$. More details can be found in Table 1 . 
Since the nonlinear pulsations can drive the star away from the equilibrium determined by the hydrostatic stellar structure equations, the final pulsation properties are obtained after a thermal relaxation time. Within a two-zone model Buchler \& Perdang (1979) have studied such thermal relaxation oscillations where the energy input comes from an underlying nuclear burning shell. Since in our case the additional energy input is due to dissipation of the kinetic wave energy within the pulsation stellar envelope, it remains to be studied whether the long term evolution $\left(t>t_{\mathrm{KH} \text {,env }}\right.$ ) will also exhibit a thermal limit cycle. Taking as the inner boundary for the envelope the radius where the velocity amplitude during an oscillation cycle becomes, e.g., less than 0.01 of the local sound velocity, we obtain the total internal energy $E_{\text {in,env }}$ of the envelope. Typically, we get $t_{\mathrm{KH} \text {,env }} \simeq E_{\text {in,env }} / L \simeq 10 \mathrm{yr}$ for the thermal (Kelvin-Helmholtz) relaxation time scale. As seen in Fig. 1, due to CPU limitations the computations have not yet been followed over such a long time scale.

\section{Conclusions}

From the number of calculations already done it seems difficult to draw definite conclusions about the long term pulsational behavior of these stars. All oscillations exhibit very fast growth rates and manifest themselves as strange modes situated in the stellar envelope. Although the overall structure of a pulsating LBV is altered by the waves traveling through the stellar envelope, the radius can either shrink or expand depending on the details of the interaction between the energy dissipation and the radiative properties of the envelope. For larger luminosity-to-mass ratios large-scale amplitudes are common in such pulsations, but no outflow has been found up to now driven by pulsations alone. Whether this is due to a lack of physical processes like rotation, dust formation and/or Doppler effects in the gas opacities which are incorporated in the present simulations must await further computations.

Acknowledgments. This work has been supported by the Österreichische Nationalbank (project 7504) and by the US NSF (project AST95-28338).

\section{References}

Buchler, J. R. \& Perdang, J. 1979, ApJ, 231, 524

Dorfi, E. A. 1998, in Proc. 27th Saas Fee Course, Numerical Radiation Hydrodynamics: Numerical Aspects and Applications (Berlin: Springer), 263

Dorfi, E. A. 1999, Journ. Comp. Appl. Math., 109, 153

Feuchtinger, M. U. 1999, A\&AS, 136, 1

Nota, A. \& Lamers, H. J. G. L. M. (ed.) 1997, ASP Conf. Ser. Vol. 120, Luminous Blue Variables: Massive Stars in Transition (San Francisco: ASP)

Yorke, H. W. 1980, A\&A, 86, 286 